\title{
A multi-center randomized, controlled, open-label trial evaluating the effects of eosinophil-guided corticosteroid-sparing therapy in hospitalised patients with COPD exacerbations - The CORTICO steroid reduction in COPD (CORTICO-COP) study protocol
}

Pradeesh Sivapalan ${ }^{1 *} \mathbb{D}$, Mia Moberg${ }^{2}$, Josefin Eklöf ${ }^{1}$, Julie Janner², Jørgen Vestbo ${ }^{3}$, Rasmus Rude Laub4, Andrea Browatzki ${ }^{5}$, Karin Armbruster ${ }^{1}$, Jon Torgny Wilcke ${ }^{1}$, Niels Seersholm¹, Ulla Møller Weinreich ${ }^{6}$, Ingrid Louise Titlestad ${ }^{7}$, Helle Frost Andreassen², Charlotte Suppli Ulrik', Uffe Bødtger ${ }^{8}$, Thyge Lynghøj Nielsen ${ }^{5}$, Ejvind Frausing Hansen ${ }^{4}$ and Jens Ulrik Stæhr Jensen ${ }^{1,9}$

\begin{abstract}
Background: The most commonly applied treatment for acute exacerbations of chronic obstructive pulmonary disease (AECOPD) is a 5-day course of high-dose systemic corticosteroids. However, this treatment has not been shown to reduce mortality and can potentially have serious side effects.

Recent research has shown that, presumably, only a subgroup of COPD patients identifieable by blood eosinophil count benefit from a rescue course of prednisolone. By applying a biomarker-guided strategy, the aim of this study is to determine whether it is possible to reduce the use of systemic corticosteroids in AECOPD without influencing the outcome.

Methods: This is an ongoing prospective multicenter randomized controlled open label trial comprising 320 patients with AECOPD recruited from four hospitals in Denmark. The patients are randomized 1:1 to either standard care or eosinophilguided corticosteroid-sparing therapy where prednisolone is not administered if the daily blood sampling reveals an eosinophil level below $0.3 \times 10^{9}$ cells/L. The primary endpoint is length of hospital stay within 14 days after recruitment. The secondary endpoints are treatment failure, 30-day mortality rate, COPD related re-admission rate, change in $\mathrm{FEV}_{1}$, and a number of adverse effect measures obtained within 3 months after the index hospitalisation date related to corticosteroid usage.

Discussion: This will be a very large RCT providing knowledge about the effectiveness of individualized biomarker-guided corticosteroid therapy in hospitalised patients with AECOPD.
\end{abstract}

Trial registration: Clinicaltrials.gov, NCT02857842, 02-august-2016. Clinicaltrialregister.eu: Classification Code: 10,010,953, 02-marts-2016.

Keywords: Systemic corticosteroids, Copd, Exacerbations, Eosinophil-guided corticosteroid-sparing therapy, Randomized controlled trial, Aecopd, Gcp, Biomarker, Length of hospital stay

\footnotetext{
* Correspondence: pradeesh.s@dadlnet.dk

'Department of Internal Medicine, Herlev and Gentofte University Hospital,

Hellerup, Denmark

Full list of author information is available at the end of the article
} 


\section{Background}

Acute exacerbation of chronic obstructive pulmonary disease (AECOPD) often leads to hospitalization and is associated with a high mortality rate. AECOPD episodes lead to accelerated decline in lung function and have negative impact on physical activity and quality of life. Furthermore, AECOPD implies high socioeconomic costs [1]. For decades, systemic corticosteroids have been a cornerstone in management of moderate to severe AECOPD. However, the optimal approach as regards the duration of treatment is unknown [2]. The REDUCE study concluded that a 5-day course of systemic corticosteroids is noninferior to 14-days of treatment [3]. Based on this, the recent GOLD strategy document recommends five to seven days of systemic corticosteroid treatment for patients with AECOPD [4].

\section{Previous research}

A more recent Cohrane review (13 studies contributed data, $n=1620$ ) regarding AECOPD patients has shown that systemic corticosteroids (compared to placebo) reduce the risk of treatment failure (number needed to treat $(\mathrm{NNT})=9 ; 95 \%$ confidence interval $(\mathrm{CI}) 7$ to 14$)$. Treatment failure was defined as patients who within 30 days required hospitalization or emergency room visits or patients requiring add-on pharmacological maintenance therapy. The risk of recurrence of AECOPD was also reduced within the first month (hazard ratio (HR) $0.78 ; 95 \%$ CI 0.63 to 0.97 ), whereas no difference in AECOPD recurrence was observed during the following 3 months [3].

Lung function measured up to $72 \mathrm{~h}$ after first administration of systemic corticosteroids showed significant improvement in forced expiratory volume in $1 \mathrm{~s}\left(\mathrm{FEV}_{1}\right)$ in the corticosteroid group (mean difference (MD) $140 \mathrm{~mL} ; 95 \%$ CI 90 to 200), whereas this difference was not observed at later time-points. No difference was observed in mortality (odds ratio (OR) 1.0; 95\% CI 0.60 to1.66). The total length of hospital stay (LOS) was shorter in the corticosteroid-treated group (MD -1.22 days; $95 \%$ CI -2.3 to -0.2 ), whereas there was no difference in the LOS in intensive care unit. The risk of steroid induced side effects was, as expected, increased (OR 2.33; 95\% CI 1.59 to 3.43) in the corticosteroidtreated group compared with the control group; number needed to harm $(\mathrm{NNH})=6 ; 95 \% \mathrm{CI} 4$ to 10$)$. Meanwhile, the proportion of side effects in the corticosteroid group (48.1\%) was considerably higher than the control group (28.5\%). The risk of hyperglycemia was increased (OR 2.79 ; $95 \%$ CI 1.86 to 4.19 ) and the absolute risk was $28.2 \%$ [3]. Others have identified serious psychiatric side effects (depression, mood changes, psychosis) and serious somatic side effects such as hypertension, ulcers, secondary adrenal insufficiency, diabetes, osteoporosis and increased risk of bone fractures in patients exposed to long-term systemic corticosteroids [5-7].

Earlier, the inflammatory process in patients with AECOPD was believed to be homogeneous, primarily neutrophilic. However, recent studies have shown that both inflammation $[8,9]$ and ethiology $[10-12]$ are heterogeneous. It has been demonstrated that a subgroup of patients with AECOPD have eosinophilic inflammation [13]. Specific attention on biological clusters and biomarkers of these have resulted in an increased understanding of the differentiated inflammatory mechanisms that exist in AECOPD [14]. Examinations of sputum from the airways in smokers have increased this understanding further [15].

A small randomized study $(n=109)$ of COPD patients with moderate exacerbations has indicated that blood eosinophil-guided corticosteroid treatment might reduce the use of systemic corticosteroids in exacerbations by $49 \%$ without simultaniously worsening of symptoms and increasing risk of treatment failure compared with standard care [16]. Furthermore, patients with initial low eosinophil count more often experienced treatment failure if they had received corticosteroid therapy rather than if they had not (15\% treatment failure in prednisolone group, $2 \%$ treatment failure in corticosteroid-saving group, $p=0.04)$. However, the study had no impact on current recommendations due to the limited sample size. Larger cohort studies documented that diagnosed COPD patients with daily symptoms have more than 3fold increased risk for AECOPD if they have an eosinophil count $\geq 0.34 \times 10^{9}$ cells/L [17].

So, the question that remains to be answered based on the currently available studies is whether the clinical effects of systemic corticosteroid treatment in hospitalised patients with AECOPD can be achieved via a more targeted and individualized eosinophil-guided treatment rather than the current "one-size fits all" treatment. To our knowledge, no randomized clinical trials have yet examined this field. This study will address this important question. The aim is to determine whether it is possible to reduce the use of systemic corticosteroids in AECOPD without influencing the outcome by applying a biomarker-guided strategy. Additionally, the study will explore whether this strategy reduces some of the most frequent side effects that occur with the current standard treatment.

\section{Methods \\ Objective}

The overall objective is to determine whether a prednisolone regimen with lower accumulated doses in hospitalised patients with AECOPD have noninferior clinical outcome than when applying standard care (SC). Based on a sufficiently sized (powered) clinical study, the aim 
is to determine if there is no relevant increase in LOS for AECOPD patients receiving eosinophil guided prednisolone-sparing therapy compared to SC. The primary endpoint of this analysis is LOS defined as the time from hospital admission to hospital discharge within 14 days after recruitment. The secondary objective is to determine if the clinical outcome for patients receiving eosinophil guided corticosteroid-sparing therapy will not be less favourable compared to SC. The following endpoints will be included when assessing the clinical outcome: 30-day mortality rate, treatment failure, COPD related re-admission rate, changes in $\mathrm{FEV}_{1}$, changes in health-related quality-of-life measured by COPD Assessment Test (CAT), changes in level of dyspnoea using the modified Medical Research Council (mMRC) Dyspnoea Scale, cumulative systemic corticosteroid dose, the period between index AECOPD and the next AECOPD and a number of adverse effect measures between index hospitalization and 3 month follow-up. The adverse effect measures include hyperglycemia, osteopenia (Bone Turnover Markers: C-terminal telopeptide of type $1 \mathrm{col}-$ lagen (CTX) and procollagen type $1 \mathrm{~N}$ propeptide (P1NP)), osteoporotic fractures, dyspepsia or ulcer complications (gastrointestinal bleeding), onset of diabetes mellitus (defined as $\mathrm{HbA} 1 \mathrm{c} \geq 48 \mathrm{mmol} / \mathrm{mol}$ ) or worsening of diabetes mellitus (defined as initiation/intensification of anti-diabetic treatment), increase in Body Mass Index and new infections treated with antibiotics since study entry.

\section{Study design, randomization and intervention}

The study will be conducted as a prospective, multicenter, randomized, controlled, open-label study in hospitalised patients with AECOPD. In total, 320 patients are expected to be included in the project within 24 months. Patients will be randomly assigned in a 1:1 fashion to either an intervention group where guidance of prednisolone treatment is based on daily eosinophil count ("eosinophil-guided corticosteroid-sparing therapy") or a standard group in which guidance of prednisolone is based on an existing guideline, see below. Pre-stratified block randomization with blocks of varying (and blinded) size will be applied to ensure equal distribution of patients on site (four different pulmonary departments in Denmark) and age (above or below 70 years of age). The study was monitored according to Good Clinical Practice (GCP) by the GCP unit at Bispebjerg University Hospital, Copenhagen, Denmark.

Patients will be randomized to one of the two treatment arms:

a) Standard Care (SC) group: Intravenous methylprednisolone $80 \mathrm{mg}$ on the first day followed by $37.5 \mathrm{mg}$ of prednisolone tablets daily for 4 days. b) Intervention group: Intravenous methylprednisolone $80 \mathrm{mg}$, followed by prednisolone tablet $37.5 \mathrm{mg}$ daily (maximum of 4 days in all) on days where the blood eosinophil count is $\geq 0.3 \times 10^{9}$ cells/L. On days with eosinophil count $<0.3 \times 10^{9}$ cells $/$ L systemic corticosteroid treatment will not be administered.

If a patient is discharged during the treatment period, a treatment based on the last measured eosinophil count will be prescribed for the remaining days within the 5 day-period.

Selection of participants All consecutive patients admitted with AECOPD in participating centres will be considered for study enrolment (Fig. 1). Fulfilling the inclusion and exclusion criteria, patients will be invited to participate in the project. The aim is to enroll a sample representative of hospitalized patients with AECOPD in Denmark. Subjects on chronic systemic corticosteroids will be eligible for inclusion if they are receiving systemic corticosteroids corresponding to 5-10 mg daily. Patients receiving non-invasive ventilation (Continuous Positive Airway Pressure/Bilevel Positive airway pressure) were only recruited if they were admitted at one of the four recruiting departments.

\section{Inclusion criteria}

- Hospitalized patients with AECOPD within $24 \mathrm{~h}$ after admission

- Age $\geq 40$ years

- Spirometry-verified COPD (defined as postbronchodilator $\mathrm{FEV}_{1} / \mathrm{FVC} \leq 0.70$ )

\section{Exclusion criteria}

- Self-reported physician diagnosed asthma

- Life expectancy less than 30 days

- Severe COPD exacerbation requiring invasive ventilation or admission to ICU

- Allergy to systemic corticosteroids

- Severe mental illness which is not controlled by medication

- People who are detained under the act on the use of coercion in psychiatry

- Severe language difficulties or inability to provide written informed consent

- Pregnancy and lactation

- Systemic fungal infections

\section{Measurements}

All outcome measures will be recorded at baseline and at the one and three month follow up visits (Table 1). 


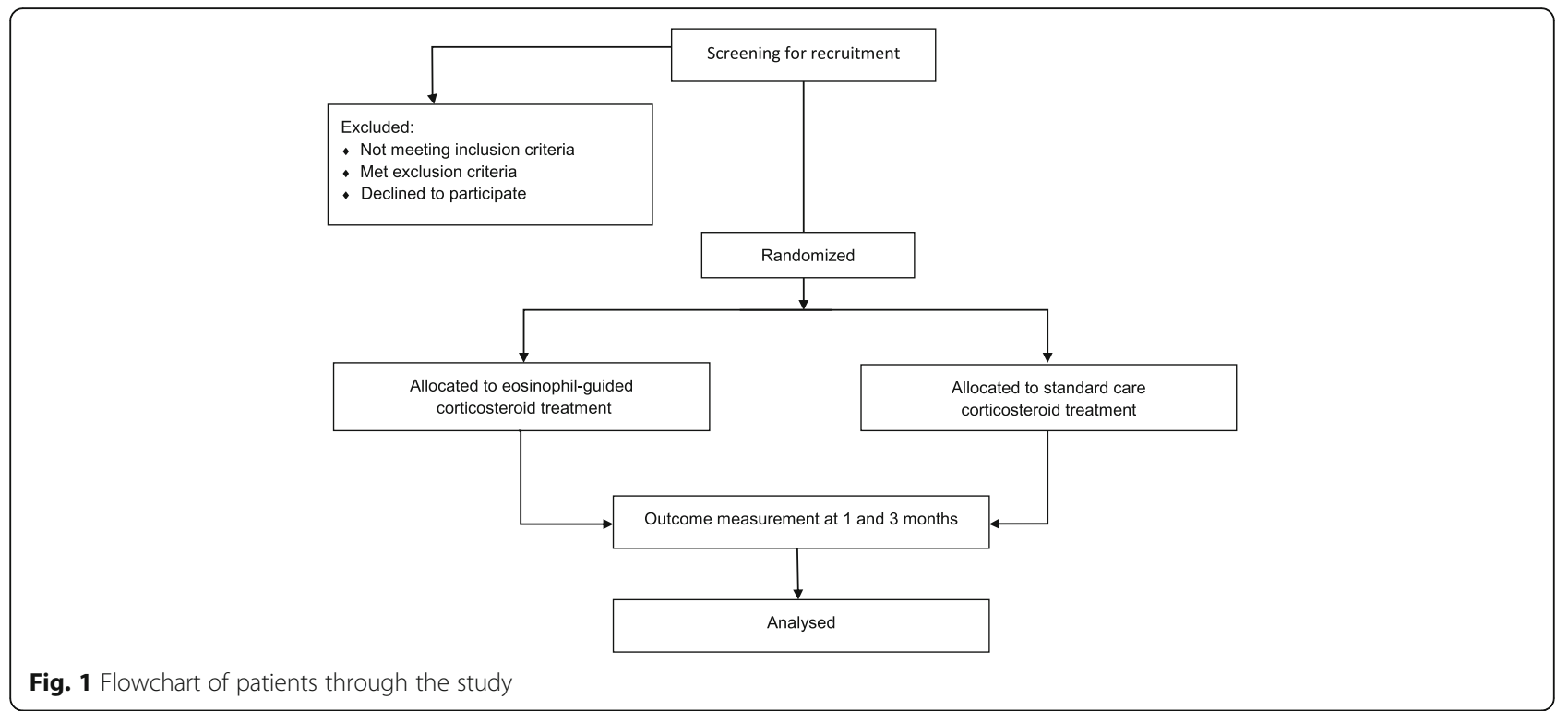

On the baseline visit (during hospitalization), the following variables will be noted: sociodemographic data (e.g. sex, age, height, weight, ethnicity), smoking history, pack-years, co-morbidities (including the presence of diabetes, heart failure, ischemic heart disease, atrial fibrillation, essential hypertension, hypercholesterolemia), employment status, support with activities of daily living at home, clinical symptoms (increased dyspnea, increased sputum volume, and increased sputum purulence (Anthonisen criteria), increased cough), disease symptoms duration, body mass index $\left(\mathrm{kg} / \mathrm{m}^{2}\right)$, number of exacerbations in the past, atopy status, mean cumulative systemic corticosteroid dose (mg) 4 weeks before study entry, use of oxygen therapy, use of noninvasive mechanical ventilation, vital signs, adverse events to systemic corticosteroids, medication adherence by day 5 , arterial blood gases and $\mathrm{Ca}^{2+}$, blood samples, findings from Chest X-ray and spirometry will be collected and stored on Case Report Forms in pseudoanonymised format at the treatment site (Table 1). Furthermore, patients will be requested to complete the CAT and mMRC Dyspnoea Scale. Follow-up visits will be conducted one and three months after discharge. If the patient does not attend the visit, the patient will be contacted for a new follow-up or - if not able to meet for an appointment at the hospital - a home visit by a doctor assigned to the project. At the one month and the three month follow up visits the following data will be assessed: BMI, HbA1c (only at the one month followup), spirometry values, CAT score, mMRC, CTX, P1NP, PTH (only at the three month follow-up), D-vitamin status (only at the three month follow-up), cumulative systemic corticosteroid dose, the period between index AECOPD and the next AECOPD, COPD related re- admission rate, dyspepsia or ulcer complications (gastrointestinal bleeding) and new infections treated with antibiotics since study entry.

Collection of data and storage will comply with Good Clinical Practice guidelines as defined by the International Conference on Harmonisation guidelines [18]. Analysis of anonymised data will be conducted by the

Table 1 Data collected at baseline and follow-up visits

\begin{tabular}{|c|c|c|c|}
\hline \multirow[t]{2}{*}{ Data collected } & \multicolumn{3}{|l|}{ Study period } \\
\hline & Baseline & 1-month & 3-month \\
\hline Demographics & $x$ & & \\
\hline $\begin{array}{l}\text { Daily blood glucose } \\
\text { measurements }\end{array}$ & $x$ & & \\
\hline $\begin{array}{l}\text { Daily leukocyte differential } \\
\text { count }^{a}\end{array}$ & $x$ & & \\
\hline $\begin{array}{l}\text { Arterial blood gases and } \\
\text { Chest X-ray }\end{array}$ & $x$ & & \\
\hline Testing for diabetes (HbA1c) & $x$ & $x$ & \\
\hline Spirometry & X (day $1+$ day 3 ) & $x$ & $x$ \\
\hline $\begin{array}{l}\text { Height measurement \& } \\
\text { vital signs }\end{array}$ & $x$ & & \\
\hline Weight measurement & $x$ & $x$ & $X$ \\
\hline Vitamin D and PTH levels & $x$ & & $X$ \\
\hline COPD Assessment Test (CAT) & $x$ & $x$ & $x$ \\
\hline $\begin{array}{l}\text { Medical Research Council } \\
\text { Dyspnoea Scale }\end{array}$ & $x$ & $x$ & $X$ \\
\hline $\begin{array}{l}\text { Bone Turnover Markers } \\
\text { (CTX, P1NP) }\end{array}$ & $x$ & $x$ & $X$ \\
\hline $\begin{array}{l}\text { Questionnaire on general } \\
\text { health condition }\end{array}$ & & $x$ & $x$ \\
\hline Pregnancy test & $x$ & & \\
\hline
\end{tabular}

${ }^{a}$ Completed by patients in the intervention group only 
principal investigator with assistance from site investigators and supervisors.

\section{Medication adherence}

All patients discharged during the treatment period ( $<5$ days) will be contacted in order to confirm whether the prescribed medication has been taken. This is to control for adherence with prescribed medication and to keep an accurate record of doses of medication for each patient.

\section{Withdrawal of study}

In general, no subject should be removed from the study for a protocol violation prior to confirmation by the coordinating investigator. A patient is only to be withdrawn from the study if the participant explicitly asks for withdrawal.

Size of the study population and statistical considerations Data will be analysed using intention-to-treat (ITT) principles, including all the data available regardless of whether the intervention is completed. The aim of the ITT analysis is also to provide unbiased comparisons among groups and avoid the effects of dropout and handle patients who deviates from the protocol, e.g. intubated patients during their hospitalization.

Patients in SC will be compared to patients with eosinophil guided prednisolone sparing therapy (Intervention group). The mean LOS $(\mu=8)$ and standard deviation $(\sigma=3.81$ days $)$ after a hospitalization for AECOPD is estimated based on previous studies comparing systemic corticosteroids versus placebo $[3,19]$. A two-sided 95\%confidence interval will be computed for the difference in LOS in the intervention group minus standard care group. We will accept a null hypothesis if the LOS in the intervention group does not exceed 1.2 days of the average LOS in the SC arm. The probability that the study will detect a treatment difference is $80 \%$ at a two-sided $5 \%$ significance level. This provides a sample size of 320 subjects. Samplesize calculation has been performed by using SAS software (version 9.4).

The results are expressed in mean (days) and SD if data is normally distributed, or as median (IQR). Data will be analysed using SAS software.

Trial Steering Committee \& Data Safety Monitoring Board A Trial Steering Committee has been established. We will meet twice a year with the Committee to advice the research team. Chair: MD, PhD Jens Ulrik Stæhr Jensen

A Data Safety Monitoring Board are in place to closely and independently monitor the safety of the CORTICOCOP study.

\section{Publication of test results}

The test results will be published regardless of whether they are positive, negative or in-conclusive. Publication in international peer-reviewed scientific journals is planned accompanied by parallel publications in Danish Medical Journal. We aim to publish in high impact scientific journals. Results that cannot be published in peer-reviewed journals will be published on www.coptrin.eu and congresses in the form of posters and oral presentations.

\section{Discussion}

COPD is a heterogeneous disease and is, by that, also associated with different responses to systemic corticosteroids for acute worsening of the disease. Although systemic corticosteroids improve some clinical outcomes in AECOPD - such as LOS, $\mathrm{FEV}_{1}$ on day 3, treatment failure and re-exacerbation rate the first month - the magnitude of benefit is small and probably restricted to subgroups of patients [3].

The novel aspect of this study is to investigate noninferiority of eosinophilic guided corticosteroid-sparing therapy against the current standardized treatment of 5 days to all AECOPD patients. The study investigates whether the accumulated dose of systemic corticosteroid treatment during admissions for AECOPD can be reduced, including the presumed side effects, while simultaneously attaining the optimal treatment effect. Studies have shown that systemic corticosteroids improve outcome in exacerbations associated with either serum eosinophil counts equal to or above $2 \%$ [16] or eosinophil counts equal to or above $0.3410^{9}$ cells/L [20]. However, on the other hand, this therapy might be detrimental in those with lower eosinophil counts [16]. A recent retrospective study has found that blood eosinophilpositive (eosinophilia $\geq 2 \%$ ) severe AECOPD require a lower daily dose of systemic corticosteroids during hospitalization compared to blood eosinophil-negative (eosinophilia <2\%) AECOPD. Thereby, the study concludes that the group with blood eosinophil-positive severe AECOPD respond better to systemic corticosteroids. Moreover, the former group had a shorter LOS (geometric mean $8.9 \pm 1.5$ versus $11.3 \pm 1.5$ days, respectively; $p=0.028$ ) [21]. The result was supported by another sub-group analysis within a large randomized clinical trial which found shorter LOS in the group with eosinophilic (blood eosinophil count $\geq 200$ cells $/ \mu \mathrm{L}$ and/or $\geq 2 \%$ of the total leukocyte count) associated exacerbations (mean (range) $5.0(1-19)$ vs. $6.5(1-33), p=0.015)$ following treatment with systemic corticosteroids [22].

Blood eosinophil count appears to be a promising biomarker that can be applied in the clinic to predict treatment response in patients with AECOPD. This could be beneficial by improving clinical outcome, decrease side effects and reduce any overuse of systemic corticosteroids [21]. The study will contribute with data to make clinical decisions on how to rationally individualize prednisolone treatment in hospitalized patients with AECOPD with the aim of optimizing treatment effect and reducing side effects. 


\section{Trial status}

\section{Patient recruitment commenced in August 2016 and is ongoing.}

\section{Abbreviations}

AECOPD: Acute exacerbation of Chronic obstructive pulmonary disease; CAT: COPD Assessment Test; COPD: Chronic obstructive pulmonary disease; CRF: Case Report Form; FEV 1 : Forced expiratory volumen in 1. Second; FEV1/ FVC ratio: also called Tiffeneau-index; FVC: Forced vital capacity;" GCP: Good Clinical Practice; GOLD: Global initiative for Chronic Obstructive Lung Disease; mMRC: Modified Medical Research Council; SC: Standard care

\section{Acknowledgements}

We are grateful for the work performed by project nurse Svenja Andersen and medical student Freja Stæhr Holm. Many thanks also to all the involved departments at the Capital Region of Denmark for allowing us to recruit patients. Thanks to the Steering Committee for all the advice provided.

\section{Funding}

The study is financed by a grant from Danish Regions (Regionernes Medicinpulje) and The Danish Council for Independent Research and by the participating sites.

\section{Availability of data and materials}

The data form the CORTICO-COP study will be available once the study is completed. Applications for data require a formal application and will be decided upon by the board of the CORTICO-COP study group.

\section{Authors' contributions}

PS prepared the study design and conducted the first draft protocol in Danish in collaboration with JU. When approved by the co-authors, PS translated the protocol into English. PS applied for approvals at the Ethics Committee, Danish Medicines Agency and the Danish Data Protection Agency. PS and JU applied for grants at Danish Regions (Regionernes Medicinpulje) and at the Danish Council for Independent Research. PS registered the trial at clinicalregister.eu and clinicaltrials.gov. All authors have read and approved the final manuscript.

\section{Ethics approval and consent to participate}

The study will be carried out to include the protection of human subjects according to the 2008 Declaration of Helsinki and in accordance with Good Clinical Practice Guidelines. Patients will be informed about the study on admission day 1 and included if accepting to participate. A completed patient informed consent form is required from all patients participating in the study and must be signed by the patient and the informing physician. The study has been approved by the Ethics Committees of all participating sides (H-15012207) and the Danish Medicines Agency (EudraCT no: 201,500,344,126) and the Danish Data Protection Agency (HGH-2015-038 and I-Suite number 04014).

\section{Consent for publication}

Not applicable

\section{Competing interests}

The authors declare that they have no competing interests.

\section{Publisher's Note}

Springer Nature remains neutral with regard to jurisdictional claims in published maps and institutional affiliations.

\footnotetext{
Author details

'Department of Internal Medicine, Herlev and Gentofte University Hospital, Hellerup, Denmark. ${ }^{2}$ Department of Respiratory Medicine, Bispebjerg University Hospital, Copenhagen, Denmark. ${ }^{3}$ Division of Infection, Inflammation and Respiratory Medicine, University of Manchester Manchester, UK. ${ }^{4}$ Department of Pulmonary Medicine, Hvidovre University Hospital, Hvidovre, Denmark. ${ }^{5}$ Department of Pulmonary and Infectious Diseases, North Zealand University Hospital, Hilleroed, Denmark. ${ }^{6}$ Department of Respiratory Diseases, Aalborg University Hospital, Aalborg, Denmark. ${ }^{7}$ Department of Respiratory Medicine, Odense University Hospital, University of Southern Denmark, Odense, Denmark. ${ }^{8}$ Department of Respiratory
}

Medicine, Naestved University Hospital, Naestved, Denmark. ${ }^{9} \mathrm{CHIP}$, Department of Infectious Diseases, University of Copenhagen, Rigshospitalet, Finsencentret, Copenhagen, Denmark.

Received: 30 January 2017 Accepted: 8 August 2017

Published online: 15 August 2017

\section{References}

1. Wang Q, Bourbeau J. Outcomes and health-related quality of life following hospitalization for an acute exacerbation of COPD. Respirology. 2005;10(3):334-40.

2. Cheng $T$, Gong Y, Guo Y, Cheng Q, Zhou M, Shi G, Wan H. Systemic corticosteroid for COPD exacerbations, whether the higher dose is better? A meta-analysis of randomized controlled trials. Clin Respir J. 2013;7(4):305-18.

3. Walters JA, Tan DJ, White CJ, Gibson PG, Wood-Baker R, Walters EH. Systemic corticosteroids for acute exacerbations of chronic obstructive pulmonary disease. Cochrane Database Syst Rev. 2014;9:CD001288.

4. GOLD: Global strategy for the diagnosis, management, and prevention of chronic obstructive pulmonary disease In.; 2017.

5. Kanis JA, Johansson H, Oden A, Johnell O, de Laet C, Melton IL, Tenenhouse A, Reeve J, Silman AJ, Pols HA, et al. A meta-analysis of prior corticosteroid use and fracture risk. J Bone Miner Res. 2004;19(6):893-9.

6. McEvoy CE, Ensrud KE, Bender E, Genant HK, Yu W, Griffith JM, Niewoehner DE. Association between corticosteroid use and vertebral fractures in older men with chronic obstructive pulmonary disease. Am J Respir Crit Care Med. 1998;157(3 Pt 1):704-9.

7. McEvoy CE, Niewoehner DE. Adverse effects of corticosteroid therapy for COPD. A critical review. Chest. 1997;111(3):732-43.

8. Bhowmik A, Seemungal TA, Sapsford RJ, Wedzicha JA. Relation of sputum inflammatory markers to symptoms and lung function changes in COPD exacerbations. Thorax. 2000;55(2):114-20.

9. Saetta M, Di Stefano A, Maestrelli P, Turato G, Ruggieri MP, Roggeri A, Calcagni P, Mapp CE, Ciaccia A, Fabbri LM. Airway eosinophilia in chronic bronchitis during exacerbations. Am J Respir Crit Care Med. 1994;150(6 Pt 1):1646-52

10. Papi A, Bellettato CM, Braccioni F, Romagnoli M, Casolari P, Caramori G, Fabbri LM, Johnston SL. Infections and airway inflammation in chronic obstructive pulmonary disease severe exacerbations. Am J Respir Crit Care Med. 2006:173(10):1114-21.

11. Seemungal T, Harper-Owen R, Bhowmik A, Moric I, Sanderson G, Message S, Maccallum P, Meade TW, Jeffries DJ, Johnston SL, et al. Respiratory viruses, symptoms, and inflammatory markers in acute exacerbations and stable chronic obstructive pulmonary disease. Am J Respir Crit Care Med. 2001; 164(9):1618-23.

12. Sethi S, Murphy TF. Infection in the pathogenesis and course of chronic obstructive pulmonary disease. N Engl J Med. 2008;359(22):2355-65.

13. Saha S, Brightling CE. Eosinophilic airway inflammation in COPD. Int J Chron Obstruct Pulmon Dis. 2006;1(1):39-47.

14. Bafadhel M, McKenna S, Terry S, Mistry V, Reid C, Haldar P, McCormick M, Haldar K, Kebadze T, Duvoix A, et al. Acute exacerbations of chronic obstructive pulmonary disease: identification of biologic clusters and their biomarkers. Am J Respir Crit Care Med. 2011;184(6):662-71.

15. Pizzichini E, Pizzichini MM, Gibson P, Parameswaran K, Gleich GJ, Berman L, Dolovich J, Hargreave FE. Sputum eosinophilia predicts benefit from prednisone in smokers with chronic obstructive bronchitis. Am J Respir Crit Care Med. 1998;158(5 Pt 1):1511-7.

16. Bafadhel M, McKenna S, Terry S, Mistry V, Pancholi M, Venge P, Lomas DA, Barer MR, Johnston SL, Pavord ID, et al. Blood eosinophils to direct corticosteroid treatment of exacerbations of chronic obstructive pulmonary disease: a randomized placebo-controlled trial. Am J Respir Crit Care Med. 2012:186(1):48-55

17. Vedel-Krogh S, Nielsen SF, Lange P, Vestbo J, Nordestgaard BG. Blood Eosinophils and exacerbations in COPD: the Copenhagen general population study. Am J Respir Crit Care Med. 2015;

18. [http://www.ich.org/fileadmin/Public_Web_Site/ICH_Products/Guidelines/ Efficacy/E6/E6_R1_Guideline.pdf].

19. Walters JA, Tan DJ, White CJ, Wood-Baker R. Different durations of corticosteroid therapy for exacerbations of chronic obstructive pulmonary disease. Cochrane Database Syst Rev. 2014;12:CD006897.

20. Vedel-Krogh S, Nielsen SF, Lange P, Vestbo J, Nordestgaard BG. Blood Eosinophils and exacerbations in chronic obstructive pulmonary disease. The Copenhagen general population study. Am J Respir Crit Care Med. 2016;193(9):965-74. 
21. Serafino-Agrusa L, Scichilone N, Spatafora M, Battaglia S. Blood eosinophils and treatment response in hospitalized exacerbations of chronic obstructive pulmonary disease: a case-control study. Pulm Pharmacol Ther. 2016;37:89-94.

22. Bafadhel M, Greening NJ, Harvey-Dunstan TC, Williams JE, Morgan MD, Brightling CE, Hussain SF, Pavord ID, Singh SJ, Steiner MC. Blood Eosinophils and outcomes in severe hospitalized exacerbations of COPD. Chest. 2016; 150(2):320-8.

Submit your next manuscript to BioMed Central and we will help you at every step:

- We accept pre-submission inquiries

- Our selector tool helps you to find the most relevant journal

- We provide round the clock customer support

- Convenient online submission

- Thorough peer review

- Inclusion in PubMed and all major indexing services

- Maximum visibility for your research

Submit your manuscript at www.biomedcentral.com/submit 